\title{
On using flood-excess volume in flood mitigation, exemplified for the River Aire Boxing Day Flood of 2015
}

\author{
O. Bokhove ${ }^{1,2} \dagger$ M.A. Kelmanson ${ }^{1}$ and T. Kent ${ }^{1}$ \\ ${ }^{1}$ Department of Applied Mathematics, University of Leeds, Leeds, LS2 9JT, UK \\ ${ }^{2}$ water@leeds, University of Leeds, Leeds, LS2 9JT, UK
}

(Received xx; revised $\mathrm{xx}$; accepted $\mathrm{xx}$ )

The goals of this paper are threefold, namely to: (i) define the rarely used concept of flood-excess volume (FEV) as the flood volume above a chosen river-level threshold of flooding; (ii) show how to estimate FEV for the Boxing Day Flood of 2015 of the River Aire; and, (iii) analyse the use of FEV in evaluating a hypothetical flood-alleviation scheme $\left(\mathrm{FASII}^{+}\right.$) for the River Aire, largely based on the actual Leeds' Flood-Alleviation Scheme II (FASII). Techniques employed are data analysis combined with general river hydraulics and estimation using bounds. By expressing FEV equivalently in terms of a square lake with a certain side-length and depth (of one to a few metres), with the same capacity, it becomes easy to visualise its dimensions and compare it with those of the river valley considered.

FEV analysis provides cost-effective estimates of new flood-mitigation measures, either prior to or in tandem with more detailed hydrodynamic numerical and laboratory modelling of river flows. It is used to illuminate five different scenarios of flood mitigation for our new $\mathrm{FASII}^{+}$, with each scenario involving a combination of higher (than existing) flood-defence walls and enhanced flood-plain storage sites both closer to and further upstream of Leeds. An integral part of this approach is a cost-benefit analysis. For policy makers, a further advantage of FEV is that it can be used to analyse and choose between flood-mitigation measures in a direct and visual manner, thereby offering better prospects of being understood by a wide, particularly non-technical, audience and city-council planning departments.

\section{Key words:}

\section{Introduction}

Precipitation records show that, whilst annual rainfall has remained roughly constant in the United Kingdom (UK) in recent decades, within that period there has been an increase and decrease in, respectively, winter and summer rainfalls. The spatial and temporal variability of precipitation is also changing. Moreover, though the amount of summer rainfall has decreased overall, it has intensified. The frequency and intensity of extreme rainfall events has increased, with similar trends observed globally in the extratropical latitudes (IPCC 2013). Heavy rainfall can have disastrous impacts via floods. Estimated costs of the 2015/16 winter flooding in the UK alone are between

$\dagger$ Email address for correspondence: o.bokhove@leeds.ac.uk 


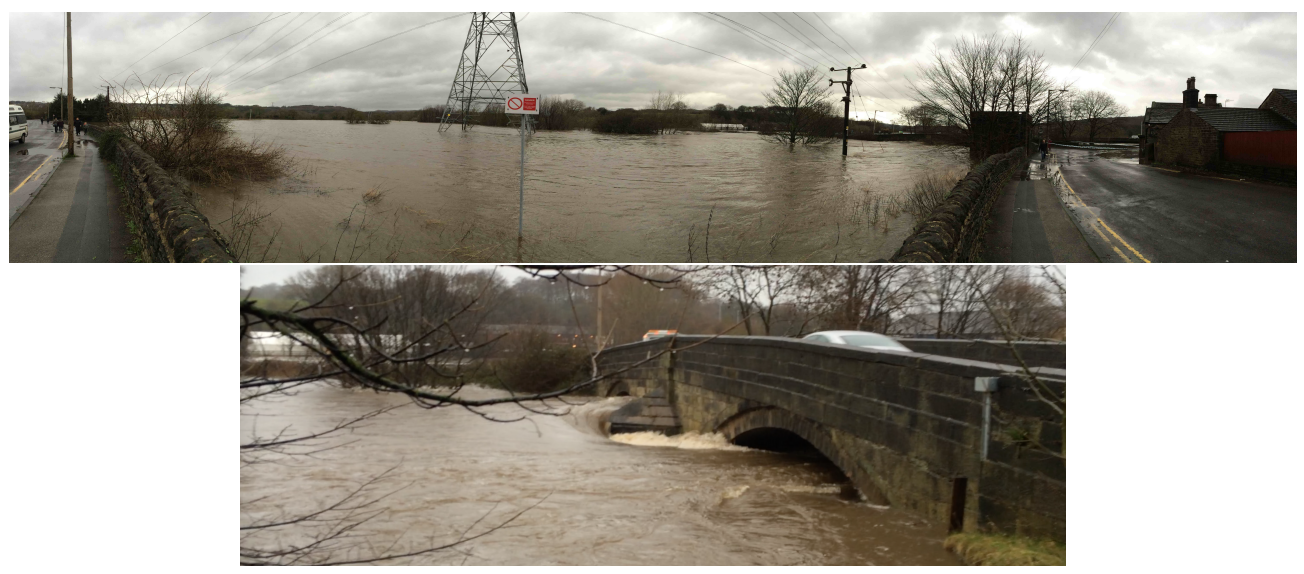

Figure 1. Boxing Day 2015 flooding levels of the River Aire near Apperley Bridge. Top: the filled flood plain upstream of the bridge constriction at 09:56:04 on 26-12-2015. Bottom: the flow under Apperley Bridge at circa 09:58:30 on the same date, showing the dangerous proximity to choking, akin to experiments in Akers \& Bokhove (2005). Photos and video cut courtesy of O.B.

$£ 1300 \mathrm{M}$ and $£ 1900 \mathrm{M}$ (Environment Agency 2018). Flooding is characterized as either fluvial (river floods due to substantial large-scale rainfall typical of winter) or pluvial (surface water or flash floods due to intense but localized rainfall typical of summer). An increase of both fluvial and pluvial flooding has been observed both in the UK and globally (Sanderson 2010; IPCC 2013), highlighting the need for effective flood-mitigation measures. Such measures are generally engineering-based (e.g., storage reservoirs, defence walls) or nature-based (e.g., catchment-wide tree planting); a suite of these different measures typically constitutes a catchment- or city-wide flood-mitigation scheme.

This paper introduces the modest yet powerful concept of flood-excess volume (FEV) to analyse a flood event in a simple way, with a view to assisting decision-making on flood-mitigation measures. In particular, it quantifies the size of a flood event and can be used to quickly and concisely partition the effectiveness of individual measures in a larger flood-mitigation scheme. To demonstrate this concept, we focus here on the socalled "Boxing Day Flood" in 2015 of the River Aire in Yorkshire, UK, due not only to its national publicity at the time, but also its proximity to the authors and concomitant familiarity, availability of data, and interest to practitioners of an ongoing county-wide mitigation scheme. A brief description of the river and flood event follows.

The Boxing Day floods occurred on $26^{\text {th }}$ and $27^{\text {th }}$ December 2015 in Yorkshire, UK, primarily affecting the River Aire and River Calder, and the severity of the flooding merited high-profile coverage in diverse national media (see, e.g., The Guardian (2015)). The Aire originates in the Yorkshire Dales and flows roughly eastwards to merge with the Ouse and Humber rivers before finally flowing into the North Sea via the Humber estuary. There was severe flooding, particularly in and around Leeds, see Fig. 1, with numerous river-level gauges exceeding previous records. For example, flood levels recorded on gauges at Armley (circa $2 \mathrm{~km}$ upstream of Leeds city centre) and Kildwick (circa $42 \mathrm{~km}$ upstream of Leeds) reached record and near-record highs of $5.214 \mathrm{~m}$ and $4.219 \mathrm{~m}$ respectively; for comparison, the previous recent highs were $4.025 \mathrm{~m}$ and $4.222 \mathrm{~m}$, respectively, in the Autumn of 2000 (Environment Agency 2016) $\dagger$. The flood resulted from record rainfall in

$\dagger$ It should be noted that the 1866 flood in Leeds was higher than the previous electronically recorded peak level before 2015. However, differences in the degree of urbanisation and record-keeping make a comparison between the 1866 and 2015 floods challenging, despite the 
the Aire catchment $(93.6 \mathrm{~mm}$ at Bingley and $69.4 \mathrm{~mm}$ at Bradford over the 48 hours from 09:00:00 on 25-12-2015 to 09:00:00 on 27-12-2015), exacerbated by high levels of saturation due to rainfall in November 2015 being the second highest on record (Environment Agency 2016). Damage estimates to date are circa $£ 500 \mathrm{M}$ for the Boxing Day 2015 floods of the River Aire, River Calder and River Wharfe combined (West Yorkshire 2016): "Over 4,000 homes and almost 2,000 businesses were flooded with the economic cost to the City Region being over half a billion pounds, and the subsequent rise in river levels allowed little time for communities to prepare."

Flooding events are generally classified in terms of their average or statistical return periods. Given a record of river-level measurements, usually as daily maxima, one can organise these data as river levels returning on average 1:10, 1:25, 1:100 or 1:200 years, etc., provided that the data set is sufficiently long. Recall that a 1:100-year return period does not mean that, after such a 1:100-year flood event, it will take 100 years before another flood of such magnitude occurs. What it does mean is that the average time between floods of that magnitude is anticipated to be 100 years. Alternatively expressed, there is a $1 \%$ chance that there will be flood of such magnitude on one day of a given year. The Boxing Day 2015 flood was an extreme event, falling outside the range of data records, and its estimated return period for the River Aire at Armley is approximately 1:200+ years (Environment Agency 2016) $\dagger$.

Recorded river levels are used mainly when comparing river-flood events, but once certain critical river-level thresholds have been surpassed, these levels convey neither the duration nor the volume of the flood. A narrow flood peak with high river levels for less than a few hours leads to a different flood event than a lower but broad flood peak with sufficiently high river levels for more than a day; the former and latter events moreover require different mitigation strategies. Information about the size of a flood is contained in rating curves, which are used to transform river-level data into discharge data (discharge is the volume of through-flow per second). These rating curves tend to be phenomenologically determined, either using velocity measurements of a river crosssection at different flow levels or via theoretical fitting based on laboratory measurements. If one integrates these discharge rates over time, one obtains a flood volume.

For example, the total water volume involved in the 2017 floods in and around Houston, Texas, which lasted for a few days following several days of extreme rainfall, has been visualised as that of a cube with sides two miles long (Business Insider 2017). Other estimates of flood volumes have been effected using comparisons in terms of doubledecker buses, football pitches, etc.: though such volumes can be and have been expressed in terms of cubic metres or miles, visualisations based on them are not necessarily helpful in placing them in the context of the valley landscape in which the rivers flow. In order to contextualise the 2017 Houston flood volume, consider now the Amazon River, which has an average flow rate of $2.09 \times 10^{5} \mathrm{~m}^{3} / \mathrm{s}$; its lower-reach width, before becoming an estuary, is $2 \mathrm{~km}$ (estimated using Google maps and Wikipedia). Therefore the above-mentioned "Houston cube" of volume $8 \mathrm{mi}^{3}$ would fill in about only two days given the Amazonian

noticeable $(\sim 0.5 \mathrm{~m})$ difference between peak levels evident in the top-left photograph in Fig. 2 of the plaques at the Armley Mills Industrial Museum. The Environment Agency estimated (personal communication by O.B.) that the 1866 flood volume was lower, with possibly slightly less rainfall.

$\dagger$ Such estimates beyond data records are possible via extreme value theory (Coles 2001), a branch of statistics which provides the mathematical framework to elegantly model the upper tail of distributions. 


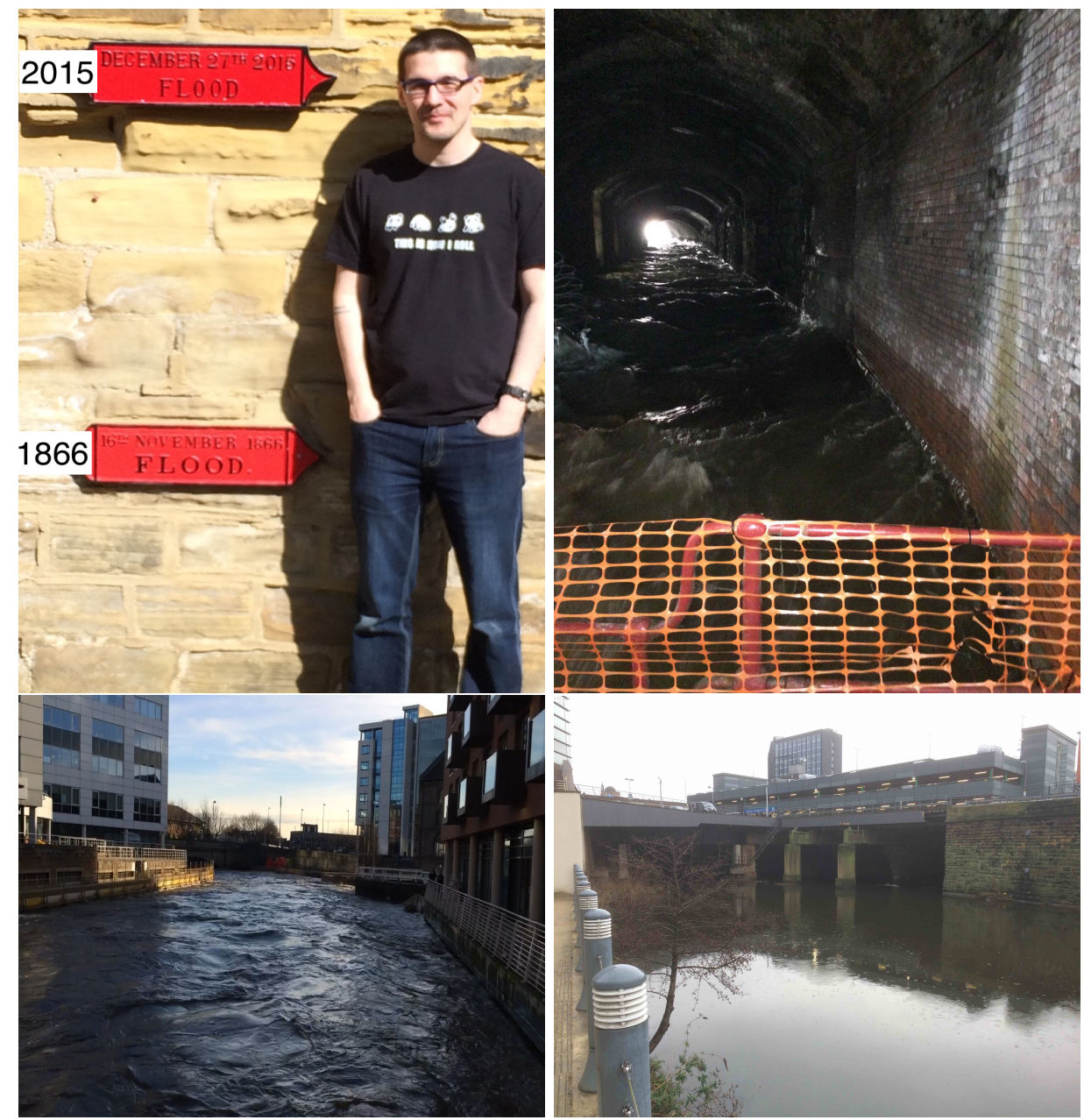

Figure 2. Top left: plaques at Leeds' Armley Mills Museum indicate the 1866 and the 2015 floodwater peak levels, the latter associated with the peak level of $5.21 \mathrm{~m}$ at the current Armley river-level gauge. Craig Duguid (Fluid Dynamics' Centre for Doctoral Training, Leeds) provides a sense of scale. Top right: high level and supercritical flow in the "Dark Arches" underneath Leeds train station at 13:36:04 on 27-12-2015; note the high-water mark on the wall outlining the peak flood level at 13:36:36 on 27-12-2015. Bottom left: flow emanating from the Dark Arches at circa 13:29:42 on 27-12-2015; the flow has become subcritical again. Bottom right: entrance and top of the weir at the Dark Arches on 19-02-2018 with subcritical inflow. Photos and video cut courtesy of O.B.

flow rate; yet the Amazon is not in flood $\ddagger$ under this average flow simply because the Amazon valley is much wider.

The above discussion on river flooding leads to the following considerations. A useful complementary concept to describe river-flood events, in addition to flood-peak levels, is that of a flood-excess volume (FEV), which is the volume of the total river discharge causing the flooding above a certain threshold river level, $h_{T}$ say, indicated in a typical flood-discharge hydrograph; see, e.g., the hatched "area" in Fig. 3. While the concept of FEV has recently (October 2017) been added to the Environment Agency modelling

$\ddagger$ Flooding in the Amazon River follows an annual "monomodal flood cycle" which is fairly predictable because it is closely related to the main rainfall season, $c f$. Trigg et al. (2012). 


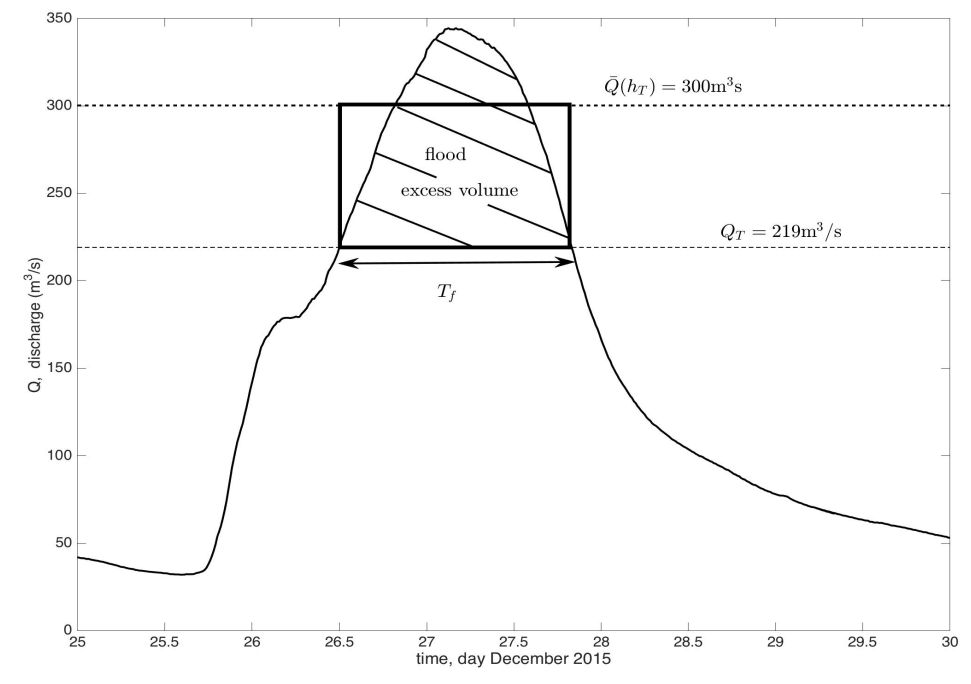

FIGURE 3. The flood-excess volume (FEV) is found by determining the hatched "area", as in (2.4), under the discharge curve $Q(t)=Q(\bar{h})=Q(\bar{h}(t))$ displayed on the vertical axis as a function of time $t$ on the horizontal axis. It involves the in-situ river level $\bar{h}=\bar{h}(t)$ as a function of time $t$ above a threshold discharge $Q_{T}=Q\left(h_{T}\right)$. Given a rating curve, $Q_{T}$ is fixed once a chosen threshold river level, here $h_{T}=3.9 \mathrm{~m}$, has been provided. The flood duration for threshold $h_{T}$ is indicated as $T_{f}=32 \mathrm{hr}$. The rectangle represents the mean (approximation) of the FEV, $V_{e} \approx\left(\bar{Q}\left(h_{T}\right)-Q_{T}\right) T_{f}=9.34 \mathrm{Mm}^{3} / \mathrm{s}$, the hatched "area" as defined in (2.3).

guidance on natural flood management (Environment Agency Oct. 2017), the concept has neither been formally defined nor explored and interpreted thoroughly to assess floodmitigation approaches and protocolst. Furthermore, once the FEV for a flood event has been estimated, it is informative to contextualise the flood in a meaningful way by visualising the volume as a cube, or a rectangular block, relative to the dimensions of the river valley concerned.

Motivated by these considerations, we present here the definition (or rather three approximations) of FEV, including the ambiguity in choosing the threshold river level $h_{T}$, the interpretation of the size of the FEV in a meaningful way, and use of the FEV in floodmitigation policy. The techniques employed are data analysis, general river hydraulics and estimation using bounds. Accordingly, the outline of the remainder of this paper is as follows: FEV is defined in $\S 2$; how to determine it is explained in $\S 3$; a full protocol for the assessment, estimation and dissemination of flood-mitigation measures is presented in $\S 4$, which is subdivided into (a) a contextual background relative to existing non-FEV protocols in $\S 4.1$, (b) a motivation for FEV using estimates based on existing scenarios with available flood-storage volume on flood plains in $\S 4.2$, and (c) an FEV-based costbenefit analysis in $\S 4.3$; a summary and discussion is found in $\S 5$, and; finally, Appendix A on natural flood management augments $\S 4.1$.

$\dagger$ O.B. defined and explored FEV in the (extant) online presentation at the Environmental Modelling in Industry Study Group at the Turing Gateway to Mathematics, Cambridge, UK,

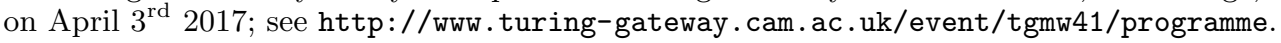




\section{Definition of flood-excess volume}

Before defining FEV, it is necessary to introduce rating curves. Gauge stations are commonly used to measure levels in rivers, across which the free-surface height generally varies little so that the measured river level can be taken as the mean $\bar{h}$ over a cross-section. The in-situ discharge $Q$ along a river cross-section can be determined by integrating a velocity profile constructed from sampled velocity measurements across the river at varying depths. Acoustic-Doppler-Current-Profilers (ADCPs) are often used for this purpose on larger rivers. By following this procedure for a range of water levels, a so-called rating curve $Q=Q(\bar{h})$, relating the measured mean river depth $\bar{h}$ to the mean discharge rate, can be established.

For several Yorkshire rivers, including the River Aire presently analysed, the Environment Agency uses a typical fit, involving numerous fitting coefficients, for the river discharge $Q=Q(\bar{h})$ as a function of the river level $\bar{h}$ (Environment Agency 2016). It reads

$$
Q=C_{j}\left(\bar{h}-a_{j}\right)^{b_{j}}, \quad j=1, \ldots, N,
$$

where coefficients $C_{j}, a_{j}$ and $b_{j}$ fit the depth data in the preordained intervals, also known as stages or limbs. We have stages $h_{j-1}<\bar{h}<h_{j}$ for $j=1, \ldots, N$. Often a limit $h_{N}$ is mentioned but for water levels above this last limit, the last limb is used for extrapolation. For $N=3$ for example, the coefficients are $a_{1}, b_{1}, C_{1}, a_{2}, b_{2}, C_{2}, a_{3}, b_{3}, C_{3}$ with depth limits $h_{0}, h_{1}, h_{2}$ and $h_{3}$. The requirement of continuity of $Q(\bar{h})$ across interval limits $\bar{h}=h_{j}$, for $j=1, \ldots, N-1$, means that only a subset of these coefficients can be independent. Additionally, for higher, extreme river levels, e.g. for $\bar{h}>h_{3}$ in the given example, there are often no velocity measurements and error bars can be high. In extreme flood conditions, the banks also overflow and the rating curves can then become (quite) different. One should bear in mind that the rating curve is approximate, especially for extreme flows where it can be an untested extrapolation. From a fluid-mechanical point of view, (2.1) is unusual (see below) because its constituents have not been a priori non-dimensionalised. As a result, $a_{j}$ has the dimension of depth $\bar{h}$ in metres, notated as $\left[a_{j}\right]=\mathrm{m}, b_{j}$ is dimensionless and $C_{j}$ is forced to have dimensions that ensure the correct $[Q]=\mathrm{m}^{3} / \mathrm{s}$ for the discharge. Hence by "unusual" above is meant that the dimension of $C_{j}$ must, by construction of (2.1), depend on the value of $b_{j}$ : specifically, one has $\left[C_{j}\right]=\mathrm{m}^{3-b_{j}} / \mathrm{s}$. Consequently, it would make more sense to write

$$
Q=\tilde{C}_{j}\left(\bar{h} / a_{j}-1\right)^{b_{j}} \quad \text { with } \quad h_{j-1}>a_{j}
$$

and to fit the dimensional parameters $\tilde{C}_{j}, a_{j}, h_{j}$ and non-dimensional parameter $b_{j}$ for all relevant $j$.

Flood-excess volume (FEV) is defined as the volume $V_{e}$ of water that caused flooding. FEV concerns the volume of river flow, at a certain location, above a certain threshold, $\bar{h}>h_{T}$, for the duration that the river is above that threshold. Here $h_{T}=0$ is in general the lowest point in the bed at the in-situ cross-sectional profile of the river. Hence, this flood duration $T_{f}=T_{f}\left(h_{T}\right)$ (expressed in time units) of a flood is then defined as the time difference of the river level breaking through the chosen threshold and dropping below this chosen threshold $h_{T}$. Given a rating curve, a graphical representation of the above definition of FEV is found in Fig. 3. We subsequently give three approximations of $\mathrm{FEV}$, because in practical situations the rating curve can be inferred only indirectly, and with varying degrees of accuracy.

The first estimate of FEV , denoted by $V_{e_{1}}$, assumes that we have an estimate of the mean discharge $\bar{Q}=\bar{Q}\left(h_{T}\right)$ (expressed in cumecs or $\mathrm{m}^{3} / \mathrm{s}$ ) during this defined period of flooding and its duration $T_{f}$, as well as the value of the discharge $Q_{T}=Q\left(h_{T}\right)$ at this 
threshold water level $h_{T}$. The FEV approximation $V_{e_{1}}$ (expressed in $\mathrm{m}^{3}$ ) is then defined as the product of the flood duration with the excess of the mean discharge over the discharge at the threshold level, i.e.,

$$
V_{e} \approx V_{e_{1}}=T_{f}\left(\bar{Q}\left(h_{T}\right)-Q_{T}\right) .
$$

Thus FEV estimate $V_{e_{1}}$ can be visualised as the "area" represented by the rectangle in Fig. 3. Formally, this FEV is equal to the total flood volume $V_{T}$ only when the threshold is zero, $h_{T}=0$, but such a low level is not an acceptable choice because, for most values of $h_{T}$, there is no flood. Most of the river water then simply flows as intended through the river channel without causing trouble. Hence, for acceptable values of $h_{T}$, the FEV will be a fraction of the total water volume flowing through the river over that same time period.

A second estimate of FEV, denoted by $V_{e}$, arises when one has river-level measurements $\bar{h}$, taken sufficiently frequently in time relative to the flood duration, as well as the rating curve $Q=Q(\bar{h})$ and possibly its error bars. Given the combination of a range of water-level measurements $\bar{h}_{k}$ during this flooding period as functions of regular times $t_{k}$, with discrete $k$ being a time index, the threshold discharge $Q_{T}=Q\left(h_{T}\right)$ and the established rating curve $Q(\bar{h})$, the discharge of the event can be determined for each threshold $h_{T}$. If the regular time interval between measurements is $\Delta t, e . g .15 \mathrm{~min}$, then FEV approximation $V_{e}$ is the sum

$$
V_{e} \approx \sum_{k=1}^{N_{m}}\left(Q\left(\bar{h}_{k}\right)-Q\left(h_{T}\right)\right) \Delta t,
$$

which approximation improves in accuracy when temporal variations in $Q$ decrease from one time interval to another, which is the case when $N_{m}$ is sufficiently large for a particular flood event. Thus FEV estimate $V_{e}$ can be visualised as the hatched "area" ( $c f$. the rectangular "area" $V_{e_{1}}$ ) in Fig. 3. This calculation of the FEV is relatively straightforward and, in the limits $\Delta t \rightarrow 0$ and $N_{m} \rightarrow \infty$, it clearly becomes the temporal integral above the threshold discharge level $Q_{T}$ and under the discharge hydrograph $Q(t)$. In essence, (2.3) is an approximation to that limiting integral form of (2.4) in which a mean water-level estimate $\overline{\bar{h}}$ (the average value of $\bar{h}$ ) has been used throughout the flood duration $T_{f}$ in combination with a mean discharge rate given by $\bar{Q}=Q(\overline{\bar{h}})$.

Our third estimate of $F E V$, denoted by $V_{e_{2}}$, is important in situations where automatic river-level measurements and rating curves are absent, while nonetheless discharge estimates are required to make flood-mitigation estimates, e.g., in local urban or remote rural areas, or in developing countriest. This third estimate is useful when only the maximum discharge $Q_{\max }$ at the peak level $h_{\max }$ is known while the relationships $Q(\bar{h})$ and $Q\left(h_{T}\right)$ are unknown. For example, when one knows a mean maximum depth $h_{\text {max }}$, the (mean) river width $W_{r}$ and maximum mean (surface) velocity $\bar{V}_{\text {max }}$, the cross-sectional area is $A=W_{r} h_{\max }$, from which an estimate for the discharge is $Q_{\max }=\bar{V}_{\max } W_{r} h_{\max }$.

Given a chosen threshold $h_{T}$, one can again estimate or establish a flood duration $T_{f}$. Assuming that we have obtained a mean water level $h_{m}$ during this flood duration, then the mean discharge $\bar{Q}$ and threshold discharge $Q_{T}$ can be estimated roughly using linear interpolations from

$$
\bar{Q} \approx \frac{h_{m}}{h_{\max }} Q_{\max } \quad \text { and } \quad Q_{T} \approx \frac{h_{T}}{h_{\max }} Q_{\max } .
$$

$\dagger$ O.B. has been asked whether a local field next to a river was adequate to mitigate a nearby village from flooding, but, without indications of mean flood-discharge rates, flood duration and the size of the tentative flood plain, such a question can hardly be addressed. 
For example, one can take $h_{m} \approx\left(h_{\max }+h_{T}\right) / 2$ only if one has the peak value $h_{\max }$ and threshold $h_{T}$. A rough estimate of (2.3) using (2.5) then gives the FEV approximation

$$
V_{e} \approx V_{e_{2}}=T_{f} \frac{Q_{\max }}{h_{\max }}\left(h_{m}-h_{T}\right) .
$$

Given that $V_{e}=V_{e}\left(h_{T}\right)$ depends on the chosen threshold $h_{T}$, it makes sense to graph $V_{e}$ as function of this somewhat arbitrary choice $h_{T}$. When $h_{T}$ equates to the maximum water level $h_{\max }$ in a particular flood event one finds, of course, that $V_{e}=0$. That case corresponds for example to a flood-alleviation measure in which flood-defence walls are raised, which consequently raises $h_{T}$ under the assumption that this barely alters the in-situ rating curve. While these three definitions of FEV (2.3), (2.4) and (2.6) are straightforward given choices of thresholds $h_{T}$, their accuracy depends on the error bars in the mean discharge $\bar{Q}$ or the rating curve $Q=Q(\bar{h})$. These error bars are often not quantified well.

\section{Using FEV for the River Aire Boxing Day 2015 flood}

The Boxing Day 2015 flood was the biggest flood on record of the River Aire in Yorkshire, UK. Leeds has three river-level gauges — operated at Kirkstall, Armley and Crown Point - for the River Aire. Data from these gauges are available online via the Environment Agency and Gaugemap (the latter at http://www.gaugemap.co.uk with easy timing and zoom-in options).

The description of the Armley gauge station includes (Environment Agency 2016): "The flow site is a velocity area station rated by a cableway spanning almost $30 \mathrm{~m}$ at the section. It is confined by the canal embankment on the right hand bank (in excess of $11 \mathrm{~m}$ high) and by a wall on the left hand bank. Bypassing is rare but can occur in the most severe conditions as shown in the December 2015 floods: water came out of the left hand bank approximately 0.8 miles upstream and travelled down Kirkstall Road towards the city centre. The channel control is from the broad-crested weir located approximately $2 \mathrm{~km}$ downstream set in the "Dark Arches" under Leeds railway station". The weir at the "Dark Arches" is the control point mentioned: it sets the upstream hydraulics at the Armley river-level gauge station. It triggers a subcritical-supercritical flow transition, with fast supercritical flow down into the "Dark Arches", back to subcritical flow. These flow conditions are exemplified in Fig. 2. We define the mean and depth-averaged river flow velocity as $u$ and the speed of surface gravity waves as the square root $\sqrt{g \bar{h}}$ of the mean depth $\bar{h}$, with gravitational acceleration $g=9.81 \mathrm{~m} / \mathrm{s}^{2}$. Technically, river flow is then defined to be subcritical when this speed $|u|$ is smaller than the speed of surface gravity waves, i.e., when $|u|<\sqrt{g \bar{h}}$, and supercritical when it is larger than this speed, i.e. when $|u|>\sqrt{g \bar{h}}$.

It is useful to estimate FEV via (2.6) which can be done quickly and without much data analysis. It can be determined from the Gaugemap data for the Boxing Day 2015 flood at Armley, taking just a threshold of $h_{T}=3.9 \mathrm{~m}$. That choice is motivated by the fact that flooding commenced at 12:17:33 on 26-12-2015, when the Armley river-level recording was at $4.16 \mathrm{~m}$; see Fig. 3. Using the slider at Gaugemap, the flood duration with levels above $h_{T}=3.9 \mathrm{~m}$ can be estimated as $T_{f}=(32 \pm 1) \mathrm{hrs}$, from 10:15am on 26/12 to $6: 15 \mathrm{pm}$ on 27/12. At 00:00:00 on 13-12, the Armley river level reached a peak of about $3.7 \mathrm{~m}$, and inspection $\dagger$ at the nearby river bank revealed that there was still circa $0.5 \mathrm{~m}$ to go before it would flow over the walled banks, which roughly matches the

$\dagger$ Personal communication with the owner of the Xfit business Kirkstall The Forge in Leeds, 
findings shown in Fig. 3. Such local knowledge can be important in setting the desired threshold. It motivates the choice of $h_{T}=3.9 \mathrm{~m}$ made above. Note that, at the time, the Environment Agency flood warnings for the Armley-Kirkstall area started for flow above $2.12 \mathrm{~m}$. Recently, in 2017 , the level for such a "first warning" was raised from $2.12 \mathrm{~m}$ to $2.70 \mathrm{~m}$. We discuss these ambiguities in choosing the threshold level $h_{T}$ : we ask if it should be, for example, in the interval $(2.7,3.9) \mathrm{m}$ or perhaps $4.16 \mathrm{~m}$, because the above observations show that it is wise to consider FEV for a range of threshold values, as will be shown later.

From the Environment Agency (2016) and the Gaugemap data, we find that $Q_{\max } \approx$ $350 \mathrm{~m}^{3} / \mathrm{s}$ with a mean water level at $h_{m} \approx 4.7 \mathrm{~m}$ based on "eye-integration" of the Gaugemap curve and a maximum level at $h_{\max }=5.21 \mathrm{~m}$, see Fig. 4 . Hence, using the above estimates one finds from (2.6) that

$$
\begin{aligned}
V_{e_{2}}\left(h_{T} \approx 3.9 \mathrm{~m}\right) & =T_{f} \frac{Q_{\max }}{h_{\max }}\left(h_{m}-h_{T}\right) \\
& =32 \times 3600 \frac{350}{5.21}(4.7-3.9) \mathrm{m}^{3}=6.2 \mathrm{Mm}^{3}
\end{aligned}
$$

This is equivalent to the capacity of a square lake with sides of length $1760 \mathrm{~m}$ (approximately $1.094 \mathrm{mi}$ ) and depth $2 \mathrm{~m}$, around which it would take approximately $80 \mathrm{~min}$ to walk. The River Aire valley between Kildwick, about $42 \mathrm{~km}$ upstream from Leeds, and Leeds ranges from $100 \mathrm{~m}$ in width to $600 \mathrm{~m}$ in width: see the planning map of the Environment Agency in that area (UK Government 2018). Now reshape the above $1760^{2} \mathrm{~m}^{2}=3.1 \mathrm{Mm}^{2}$ square lake into an equivalent-area rectangular lake of width $200 \mathrm{~m}$ and length $15.5 \mathrm{~km}$, maintaining the same depth of $2 \mathrm{~m}$. If and only if a series of (disjunct) flood-storage areas can be created of that width and cumulative length, in which the flood waters can be held and stowed up by an extra $2 \mathrm{~m}$ above the Boxing Day flood levels, then the FEV can be held at zero with corresponding river levels at Armley at or below $3.9 \mathrm{~m}$. There are various locations in the River Aire valley where extra buffer capacity may be available, to be used in rare occasions of extreme floods, on average 1:50 or 1:100 years, as further inspection of footage of the Boxing Day 2015 floods of the Aire Valley reveals, e.g. see Fig. 12 .

Since bypassing (see Fig. 12, subfigure 4) at the Armley gauge station is rare, as stated in the Armley station description quoted above, the rating curve seems relatively trustworthy even for high discharge rates. The coefficients for the rating curve (2.1) given in Table 1 are new ones from a 2016 update, which have been corrected by using flow data as well as a comparison with a hydraulic modelling curve produced by Arup, $c f$. Environment Agency (2016).

The river level and calculated flow rates based on the rating curve of the River Aire at the Armley gauge station are shown in Fig. 6. Measurement time intervals are $15 \mathrm{~min}$. The third highest peak on 15-11-2015 -about 200 days after May $1^{\text {st }}$ - is $3.709 \mathrm{~m}$ high. The second highest peak, of height $3.699 \mathrm{~m}$, occurs on 13-12-2015. From November 2015 through to March 2016, the mean river level is thus seen to remain relatively high, thereby corroborating our earlier statement that the November and December months were either the second wettest or wettest on record. An exploded view around the Boxing day peak is shown in Fig. 7, in which also the rating curve and its linear approximation are given. Additionally, a chosen threshold level of $h_{T}=3.9 \mathrm{~m}$ is indicated, via horizontal lines, along with the discharge at that level. The peak level in the record at 02:15:00 on

who O.B. had asked to check the river level on the evening of 12-12-2015 and whether his business was in danger of flooding. 


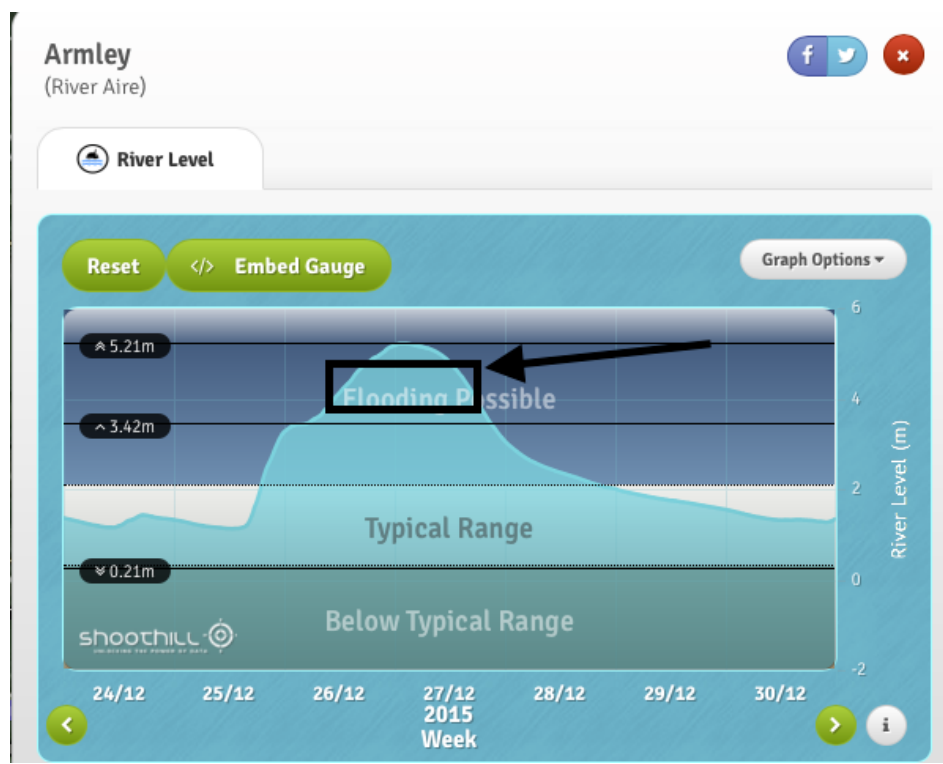

FiguRE 4. By using Gaugemap at the Armley station, one can quickly estimate the river level of the mean area indicated by the top rectangle above $h_{T}=3.9 \mathrm{~m}$, which yields a mean height at the arrow of about $4.7 \mathrm{~m}$; one can also also find the duration $T_{f} \approx 32 \mathrm{hrs}$ for which the flood level was above $h_{T}$, by using the online time slider.

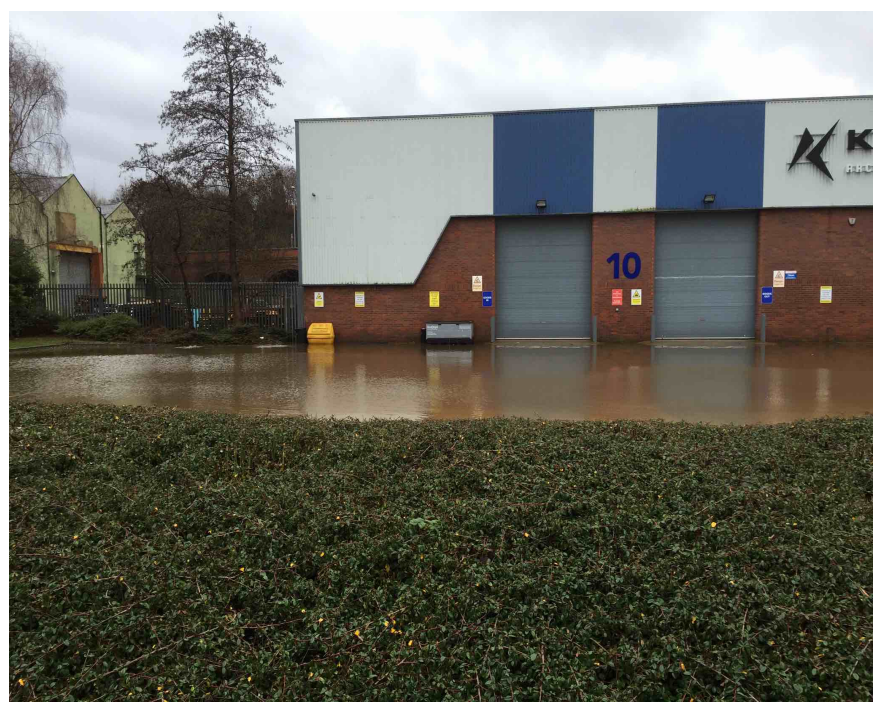

FiguRE 5. Flooding starts at Kirkstall on 26-12-2015 at 12:17:33 when the Armley river level recorded a level of $4.16 \mathrm{~m}$, which motivates one to take a threshold river level at $h_{T}=3.9 \mathrm{~m}$. Photo courtesy Jessica Worsey.

27-12-2015 was $5.217 \mathrm{~m}$ and the corresponding discharge $344 \mathrm{~m}^{3} / \mathrm{s}$. The FEV is the area indicated between the discharge at the threshold $h_{T}$ (indicated by the horizontal dashed line) and discharge rates (the solid curve). Using (2.4), the FEV integrates to

$$
V_{e} \approx(9.34 \pm 0.51) \mathrm{Mm}^{3} \approx 2150 \times 2150 \times 2 \mathrm{~m}^{3}
$$

or, equivalently, the capacity of a square lake with sides of length $2161 \mathrm{~m}$ and depth $2 \mathrm{~m}$; 


\begin{tabular}{c|c|c|c|c}
$j$ & $\begin{array}{c}h_{j} \\
\mathrm{~m}\end{array}$ & $\begin{array}{c}C_{j} \\
\mathrm{~m}^{3-b_{j}} / \mathrm{s}\end{array}$ & $\begin{array}{c}a_{j} \\
\mathrm{~m}\end{array}$ & $\begin{array}{c}b_{j} \\
-\end{array}$ \\
\hline 1 & 0.685 & 30.69 & 0.156 & 1.115 \\
2 & 1.917 & 27.884 & 0.028 & 1.462 \\
3 & 4.17 & 30.127 & 0.153 & 1.502
\end{tabular}

TABLE 1. The new (as of 2016) coefficients $C_{j}, a_{j}, b_{j}$ as well as the limb thresholds $h_{0}=0.2$ and $h_{j}$ for $j=1,2,3$ for the rating curve at the river-level gauge station at Armley in Leeds.
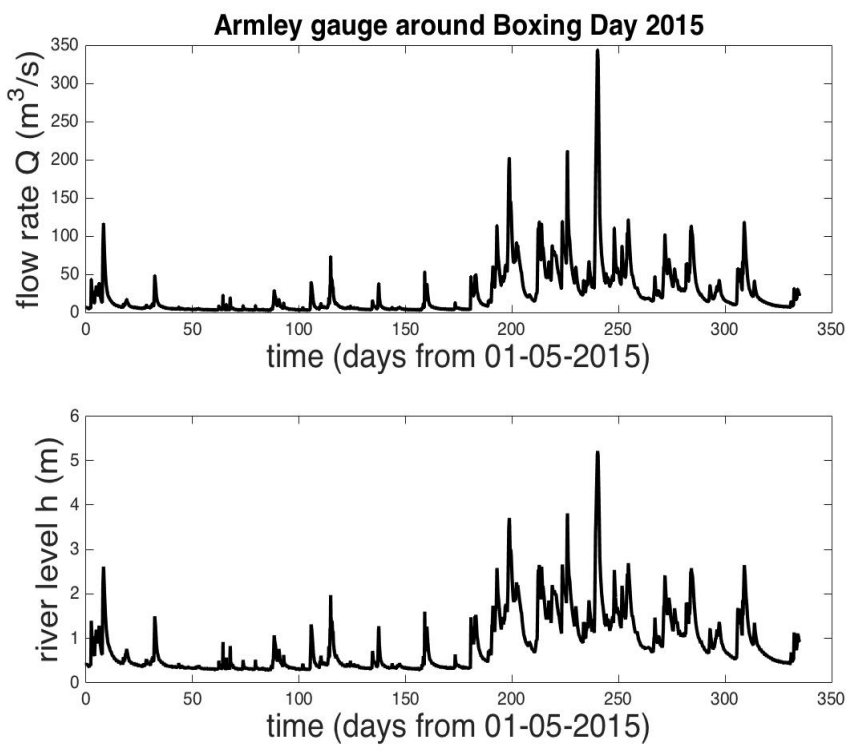

FiguRE 6. Flow rates and river levels of the River Aire at Armley, Leeds, from May 2015 until the end of March 2016.

this figure is approximately 1.5 times the crude, quick estimate in (3.1). Note that the estimate (3.1) is itself in error because the relationship between river level and discharge rate as encoded in the rating curve is nonlinear, in contrast to the linear rating-curve assumption used in the estimate.

To investigate the dependence of FEV on the chosen threshold level $h_{T}$, the calculation is repeated for a range of thresholds $h_{T} \in[2.7,5.22] \mathrm{m}$, from the level at which the Environment Agency now issues a flood warning at $2.7 \mathrm{~m}$ until the peak water level is reached. Both the excess volume and the equivalent lake size of depth $2 \mathrm{~m}$ are displayed in Fig. 8, showing that $V_{e} \in[0,25] \mathrm{Mm}^{3}$, so that the length of the sides of the $2 \mathrm{~m}$-deep square lake are in the corresponding range $[0,3500] \mathrm{m}$. The rating-curve analysis provided by the Environment Agency (Environment Agency 2016) gives an error bar within each riverlevel limb or range of $(5.42,3.44,5.28) \%$ so, to be safe, we can take as a conservative limit the error in the rating curve to be $5.5 \%$ overall. Since in the calculation of the excess volume the rating curve is used twice, via a subtraction, the cumulative error in the excess volume will remain circa $5.5 \%$, following error propagating techniques, yielding the quoted $0.51 \mathrm{Mm}^{3}$ error in $(3.2)$.

In a companion article (Bokhove et al. 2018), we also determine the FEV for the Boxing 

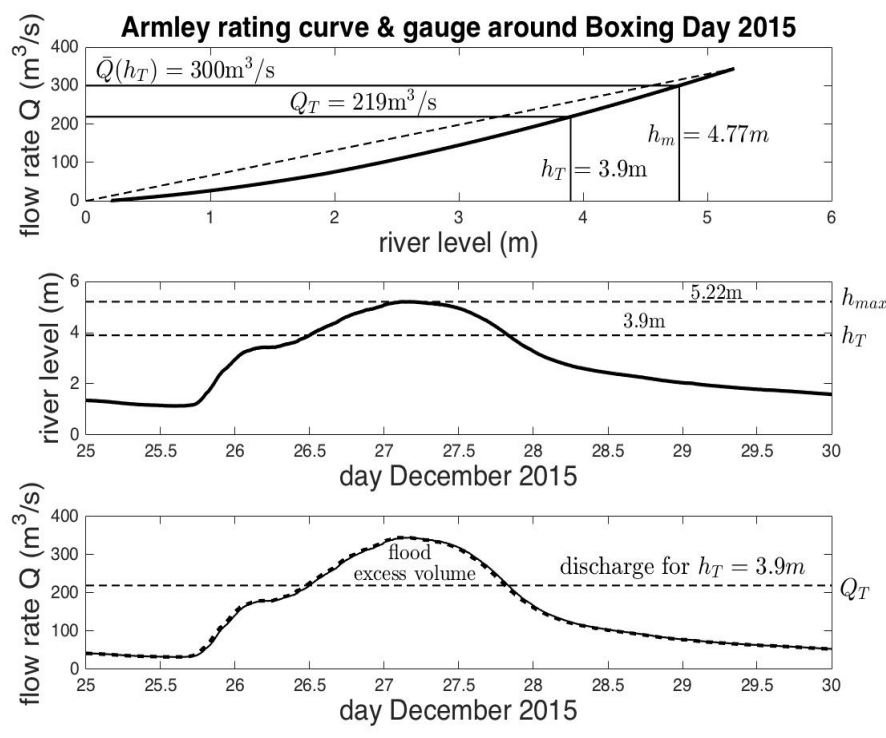

FiguRE 7 . The rating curve (solid line, top panel) and its linear approximation (dashed line) are displayed as well as exploded views on river levels (middle panel) and peak flow rates (bottom panel) of the River Aire at Armley, Leeds, around Boxing Day 2015. The dashed horizontal lines indicate a chosen threshold, the corresponding discharge and the maximum river level.
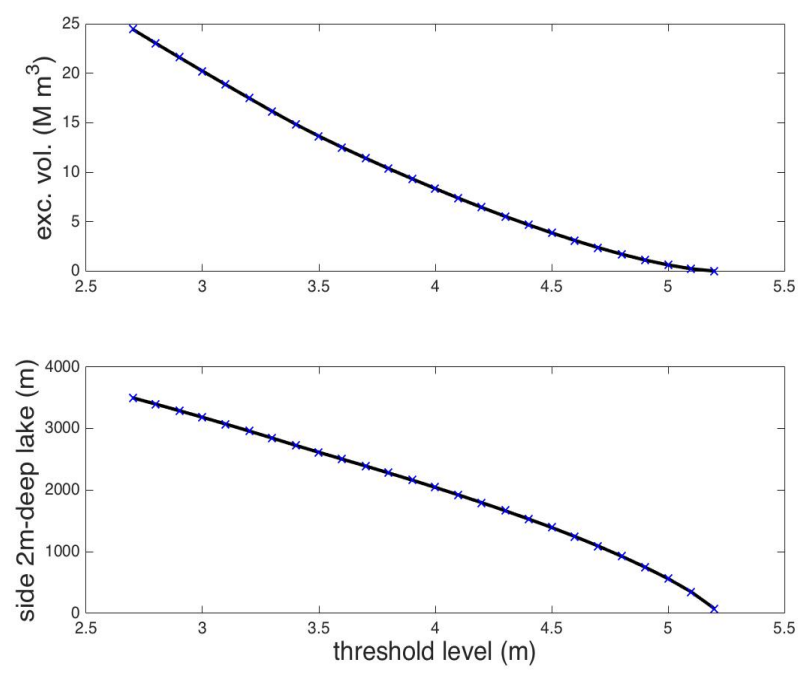

Figure 8. River Aire excess volumes $V_{e}$ (top panel) and equivalent square lake sizes (bottom panel) for various threshold levels $h_{T}$, from the Armley data of the Boxing flood 2015 flood.

Day 2015 flood of the River Calder in Mytholmroyd and for the June 2007 summer floods, of the River Don at Sheffield Hadfields, which caused widespread damage and in which three people died (The Guardian 2007). The River Calder forms the catchment south of the River Aire, flows predominantly eastwards and merges with the River Aire in Castleford. The River Don catchment lies south of the River Calder in Yorkshire. The 
following flood-excess volumes $V_{e}=(9.34 \pm 0.51,1.65 \pm 0.22,3.00 \pm 0.24) \mathrm{m}^{3}$ were found for chosen threshold levels of $h_{T}=(3.9,4.5,2.9) \mathrm{m}$ for the River Aire, River Calder and River Don, respectively.

These volumes can also be expressed as the capacity of $2 \mathrm{~m}$-deep square lakes with side-lengths of $(2161,908,1225) \mathrm{m}$ : they allow one to visualise the variety in the size of river floods. This representation of flood-excess volumes as square lakes with realistic lake depths is insightful when considering flood-mitigation strategies. Given the size of the lake as well as the width and length of a river valley, one can begin to make a ballpark estimate as to whether flood-plain enhancement for flood storage and other flood-mitigation measures will fit within a particular river catchment.

\section{FEV protocol for flood mitigation}

\subsection{Background and existing non-FEV protocols}

Following the Boxing Day floods in 2015, Leeds City Council (LCC) and the Environment Agency in Leeds (EA Leeds) have designed and proposed a Leeds FloodAlleviation Scheme II (FASII) (Leeds City Council 2018; Leeds Executive Board 2017). The information on FASII, augmented with educated guesses of flood-storage volumes and additional cost estimates, will be used to create a hypothetical flood-alleviation example, denoted by $\mathrm{FASII}^{+}$. It will be used as a pseudo-realistic case to promote the use of FEV in analysing flood-mitigation strategies. The actual FASII concerns the proposed flood protection upstream of Leeds' railway station against flood events with a 1:200year return period, while Flood-Alleviation Scheme I (FASI), completed in 2017, already protects Leeds downstream from this station against flood events with a 1:100-year return period.

Since we build our hypothetical scheme around FASII, we first provide a brief summary of FASII's essential features.

(i) The basic scheme aims to protect Leeds against flooding events with a 1:200-year return period.

(ii) Natural flood management (NFM) will be used to offset increased flood risk due to climate change. In the upper catchment of the river, NFM will include the re-meandering of the River Aire and its tributaries and the planting of trees to increase tree coverage in the catchment from $7 \%$ to $15 \%$. Further discussion of NFM and upper bounds on the flood-mitigation effects of tree planting is deferred to Appendix A.

(iii) Certain constrictions in the river course causing flow stowage at floods, recall Fig. 1, will be removed within Leeds. These constrictions include, for example, narrow river passages formed by derelict or abandoned bridge structures.

(iv) Two flood plains, approximately seven miles upstream from Leeds, one at Calverley and one at Rodley, are considered to provide flood storage. Estimated storages at Calverley and Rodley are respectively $1 \mathrm{Mm}^{3}$ and $2.2 \mathrm{Mm}^{3}$.

(v) Higher flood-defence walls will be used in Leeds, with varying heights at different locations, depending on the inclusion of: (a) only the highest flood-defence walls, higher walls with (b) the Calverley flood-storage area, (c) only the Rodley one or (d) both the Calverley and Rodley flood areas. A breakdown of the height of the defence walls of these four options is given in a table (Leeds Executive Board 2017, §3.5.1), each option giving protection against floods with a 1:200-year return period, presumably based on computer simulations of the river hydraulics in such a flood event. Some of this breakdown is reproduced in Table 2 for two locations with some of the highest proposed flood-defence walls; Table 2 has moreover been extended with extra information on the percentages 
Location $\quad$ Walls only $\mid$ plus Rodley $\mid$ plus Calverley $\mid$ plus Rodley \& Calverley

\begin{tabular}{|c|c|c|c|c|}
\hline $\begin{array}{c}\text { Whitehall } \mathrm{Rd} \text { to } \\
\text { Wellington } \mathrm{Br}\end{array}$ & $\begin{array}{c}1.74 \\
-\end{array}$ & $\begin{array}{c}1.53 \\
12.07 \%\end{array}$ & $\begin{array}{l}1.6 \\
8.05 \%\end{array}$ & $\begin{array}{c}1.5 \\
13.79 \%\end{array}$ \\
\hline $\begin{array}{l}\text { Viaduct Rd to } \\
\text { Cardigan Fields }\end{array}$ & $\begin{array}{c}2.27 \\
-\end{array}$ & $\begin{array}{c}2.0 \\
11.89 \%\end{array}$ & $\begin{array}{c}2.09 \\
7.93 \%\end{array}$ & $\begin{array}{c}1.96 \\
13.66 \%\end{array}$ \\
\hline$\%$ of FEV & 100 & 76.45 & 89.29 & 65.74 \\
\hline
\end{tabular}

TABLE 2. The height of the defence walls proposed in FASII for protection against the 1:200-year flood, along two different river stretches for the cases with only walls, walls with also the Rodley flood storage, or also the Apperley Railway Bridge/Calverley storage, or both. The wall-height reduction is given as a percentage for each location and can be compared against the extra flood-storage reduction percentage with respect to the FEV of $V_{e}=9.34 \mathrm{Mm}^{3}$ chosen.

gained from, and a comparison with, the FEV fraction alleviated based on the FEV at the Armley station of $V_{e}(3.2)$.

(vi) FASI will be updated to provide increased protection against floods; specifically, up from a 1:100- to a 1:200-year return period.

(vii) Potential flood-storage sites further upstream, at the Cononley Washlands near Skipton and Holden Park near Keighley, about 42km upstream from Leeds, which both have substantially larger flood-storage volumes, have been dismissed because they were deemed too far away from Leeds and would thus not be able to protect against flooding with extreme rainfall nearby Leeds.

(viii) The costs mentioned are $£ 109$ M (Leeds Executive Board 2017), inclusive, for the combined case of flood protection with higher flood-defence walls in Leeds and the enhanced Calverley flood-storage area. When also the Rodley area is included, the costs become $£ 123 \mathrm{M}$ (Leeds Executive Board 2017), whence the costs for the enhancement of the Rodley flood plains are deduced to be $£ 14 \mathrm{M}$.

Before proceeding with a flood-storage and cost-benefit analysis using FEV, a few comments on the above are required.

Per (i), the Boxing Day flood of 2015 was an extreme flood event with a $1: 200^{+}$. year return period. FASII, designed to protect against a flood with a 1:200-year return period, does not therefore protect against a future Boxing-Day-type flood in Leeds. A new Boxing-Day-type flood would consequently overtop the higher defence walls proposed in FASII, which moreover does not mention whether there will be sluice gates that can channel floodwaters, which have overtopped the defence walls and entered into the city, back into the river once river levels are subsiding. That is not a moot point: in April 2003, the Salado River overtopped locally lower flood-defence walls upstream of the city of Santa Fe in Argentina and caused the water elevation inside the city at a point downstream of the breached area to be $2.48 \mathrm{~m}$ higher than the flood levels in the river (Vionnet et al. 2006).

Per (ii), NFM contributes $0 \%$ to the basic 1:200-year return-period flood protection without climate-change effects being taken into account.

Per (iii), removing constrictions can alter or alters the FEV calculation at Armley but we will ignore this effect. While the $\mathrm{FEV}(3.2)$ of $V_{e}=9.34 \mathrm{Mm}^{3}$ concerns a $1: 200^{+}$-year return-period event, we will use it as the $\mathrm{FEV}$ for $\mathrm{FASII}^{+}$with its 1:200-year return period, either by ignoring the difference or by marginally reducing the threshold $h_{T}$, 
in such a way that it precisely compensates for the lowered peak flow. In engineering practice, this could be corrected by calculating the FEV corresponding to a computersimulated flood in Leeds for the target flood, or an ensemble of target floods, with a 1:200-year return period. FEV has not been used as a tool in FASII, so such an updated FEV is not available.

Per (v), building higher walls will also change the rating curve and the FEV at Armley station but we expect the changes to be small given that "overbanking" was reported to be relatively small at Armley (Environment Agency 2016). Hence, for simplicity, we keep our original FEV of $V_{e}=9.34 \mathrm{Mm}^{3}$ for also the 1:200-year return-period flood event. We can ignore the influence of $\mathrm{FASI}$ in (vi) on $\mathrm{FASII}^{+}$, given that the weir under the railway station acts as a control point, even in flood conditions, cf. Fig. 2.

Per (vii), for the Boxing Day flood in 2015, the peak flow at Kildwick was about $163 \mathrm{~m}^{3} / \mathrm{s}$ with the peak flow at Armley being $344 \mathrm{~m}^{3} / \mathrm{s}, c f$. Environment Agency (2016). A significant tentative reduction of the discharge at or near Kildwick, found by enhancing the flood-storage capacity at the Cononley Washlands near Skipton upstream of Kildwick as well as the Holden Park flood plain near Keighley, would reduce the inflow and therefore increase the flood resilience in Leeds. For the Boxing Day flood in 2015 with its peak rainfall around Bingley and Bradford downstream of Kildwick, a reduction by $26 \mathrm{Mm}^{3} / \mathrm{s}$ over $T_{f}=32 \mathrm{hrs}$, yielding $26 \times 32 \times 3600=3 \mathrm{Mm}^{3}$ in volume, would yield a roughly similar reduction at Armley and a lowering of the peak levels by $(5.217-4.958) \mathrm{m}=0.269 \mathrm{~m}$ (obtained by using the Armley river-gauge data). These river levels follow from the available measurements at Armley. (We tacitly assumed here a linear rating curve, for simplicity, but the argument can be updated using the measurements and rating curve at Kildwick.) This contrasts with the arguments of LCC in Leeds Executive Board (2017), which dismisses the upstream storage sites because they are deemed to be too far away. Morever, while the argument to dismiss flood storage further upstream is used by LCC to dismiss these upstream storage sites, it is not used to dismiss the NFM flood-alleviation measures far upstream (see Appendix A), which use of arguments appears to the authors to be inconsistent.

Per (viii), given the lack of background on further costings of FASII, we made up reasonable figures and created a hypothetical flood-alleviation scheme $\mathrm{FASII}^{+}$to illustrate our new, generic methodology and protocol to analyse flood-alleviation schemes using FEV analysis. This does imply that no inferences other than methodological ones can be drawn from what follows. Our FASII+ does not apply directly to the real FASII.

\subsection{Scenarios with available flood-storage volume on flood plains}

From the augmented Table 2, the separate reductions of the wall heights due to the inclusion of either the Calverley or Rodley flood-storage sites are circa $8 \%$ or $12 \%$. Given the FEV we adopted, this leads to available flood-storage volumes respectively of $0.08 V_{e}=0.75 \mathrm{Mm}^{3}$ and $0.12 V_{e}=1.1 \mathrm{Mm}^{3}$ for Calverley and Rodley. We contrast these reduced values with the mentioned total flood-storage volumes of $1 \mathrm{Mm}^{3}$ and $2.2 \mathrm{Mm}^{3}$ quoted in FASII. We define available flood-storage volume as the extra flood-storage volume available above and beyond the flood-storage volume already in use by here concerning the 1:200-year return-period flood event. For a flood with a lower return period, the available flood-storage volume will increase given that the reference river levels will be lower for such a (N.B. more likely) flood event. The concept of available flood-storage volume $\dagger$ is related to the concept of available potential energy, introduced

$\dagger$ It was a remark by Andy Moores of the Environment Agency at the last General Assembly in January of 2018 of the network "Maths Foresees", on flood plains already being partially 

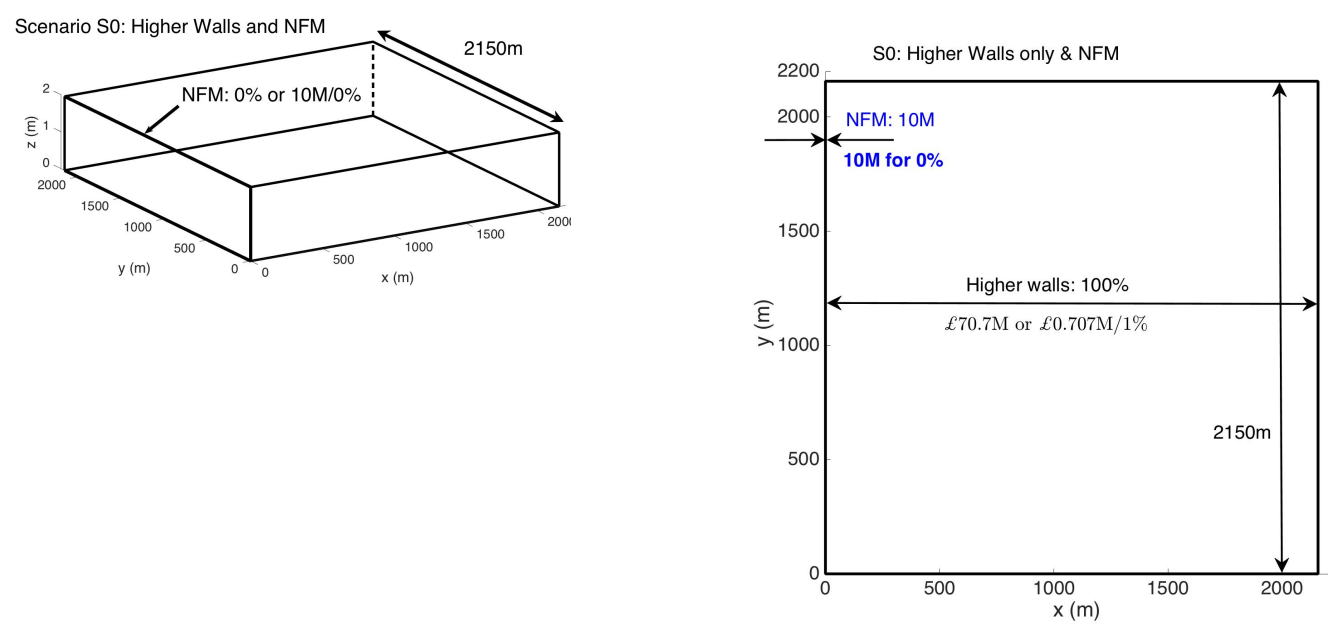

Figure 9. Left: the FEV expressed as a square 2m-deep lake of side-length $2150 \mathrm{~m}$. Right: the FEV lake, shallow relative to its side length, viewed from the top. Basic scenario S0 is depicted with higher flood-defence walls giving 100\% protection and Natural Flood Management (NFM) $0 \%$ against the desired 1:200-year return-period flood event. Indicated in the square lake are the relative size of each flood-mitigation measure, expressed both as lake area and the length of double-ended arrows, as well as its costs in UK pounds sterling. Split and total costs are indicated in absolute terms and per percentage of the total flood mitigation gained. Total costs, here equalling the protection offered by using only higher flood-defence walls, are indicated under the double-ended arrow spanning the width of the entire flood lake.

in Lorenz (1955) and used successfully in atmospheric science and oceanography; see also Shepherd (1993). Available potential energy refers to the internal and potential energy available in the atmosphere for conversion to kinetic energy: it is significantly smaller than the total internal and potential energy because it is calculated relative to a hydrostatic reference atmosphere. The reduction of volume between the total and available floodstorage volumes can be confirmed with common-sense estimates based on the size of existing flood plains in the River Aire Valley, as we will analyse next.

When one considers the potential flood plains at Cononley Washlands and Holden Park by inspection from the map in Fig. 10, a ballpark estimate of the extra flood-plain storage is $2150 \mathrm{~m} \times 350 \mathrm{~m} \times 2 \mathrm{~m}=1.5 \mathrm{Mm}^{3}$ for each site. When one uses a similar ballpark estimate for the Rodley site one gets $1000 \mathrm{~m} \times 350 \mathrm{~m} \times 2 \mathrm{~m}=0.7 \mathrm{Mm}^{3}$, which is lower by a factor of $f_{a}=1.57$ than the $1.1 \mathrm{Mm}^{3}$ found from the deduction based on Table 2 and the FEV of $V_{e}=9.34 \mathrm{Mm}^{3}$. The available flood-storage volumes of Cononley Washlands and Holden Park flood plains are estimated to be $V_{c}=f_{a} \times 1.5 \mathrm{Mm}^{3}=2.355 \mathrm{Mm}^{3}$ where we used the same factor $f_{a}$, in analogy with the appearance of this factor between the ballpark volume estimate and the calculated available storage volume for the Rodley available flood-storage volume. The combined, enhanced flood-storage volume of these flood plains then yields circa $2 V_{c} / V_{e}=2 \times 2.355 / 9.34=50.4 \%$ of the required flood protection.

occupied by flood waters, that led to our conceptualisation of available flood-plain or -storage volume. 


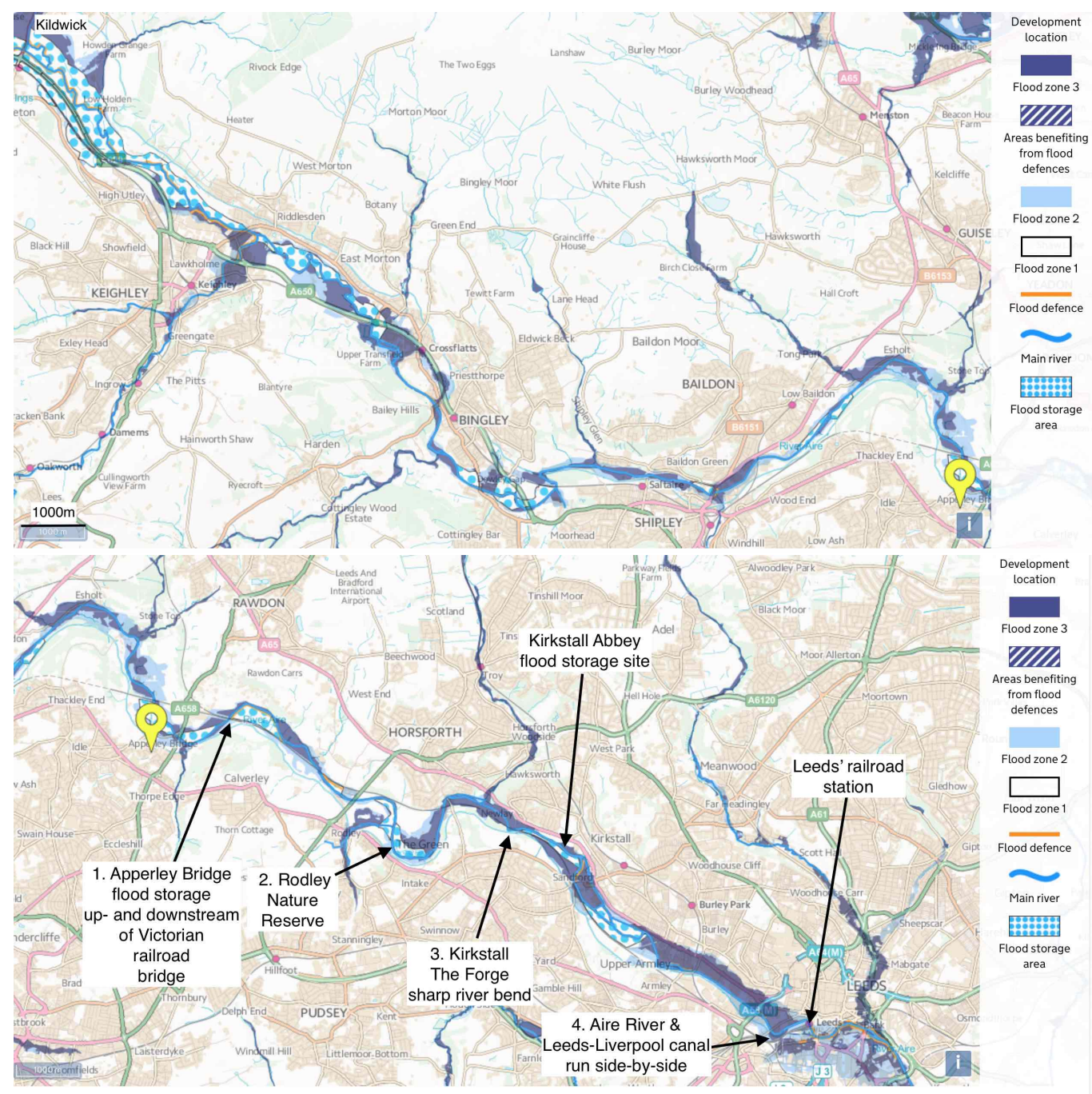

FiguRE 10. Environment Agency flood-risk maps (UK Government 2018) of the sectors Kildwick (top left in upper map) to Apperley Bridge (yellow marker in lower map) and Apperley Bridge to Leeds (bottom right in lower map). It is important to assess whether extra flood storage is available, which can be done via the marked locations 1, 2, 3 and 4 on the lower map, which respectively correspond to the top-left, top-right, bottom-left and bottom-right photographs in Fig. 12.

\subsection{FEV-based cost-benefit analysis}

Finally, to present our new diagnostic, the various costs for our hypothetical $\mathrm{FASII}^{+}$ in $\S 4.2$ are estimated and then posited to be as follows:

- Calverley's flood-storage enhancements gained by building an adjustable weir in extreme flood events will cost $£ 10 \mathrm{M}$ (including both a flood-warning system and compensation against loss of farming income), given that the (slightly larger) Rodley flood-plain enhancement costs $£ 14 \mathrm{M}$. This is approximately (see Table 2 ) $£ 10 \mathrm{M} / 8 \%=£ 1.25 \mathrm{M}$ per one percent of flood protection.

- Rodley's flood-plain enhancement is approximately $£ 14 \mathrm{M} / 12 \%=£ 1.17 \mathrm{M}$ per one percent of flood protection. 
- Calverley and Rodley's flood-plain enhancements, in combination, are approximately $£ 24 \mathrm{M} / 14 \%=£ 1.71 \mathrm{M}$ per one percent of flood protection. It is noteworthy that, given the results of the hydraulic simulations and the figures in Leeds Executive Board (2017), together with the data in Table 2, the discrepancy of $6 \%$ between the sum of the independent reductions and the total reduction of the combined sites remains unexplained.

- NFM costs are budgeted at $£ 10 \mathrm{M}$.

- FASI updates to a 1:200-year flood protection are costed at $£ 14 \mathrm{M}$.

- Constrictions to be removed are costed at $£ 10 \mathrm{M}$.

- Higher defence-wall costs are deduced from the above costings and the stated total cost of FASII of $£ 109 \mathrm{M}$, for higher walls and the Calverley flood-storage inclusive; each has a respective relative percentage of flood protection of $92 \%$ and $8 \%$, hence the costs of the case with $100 \%$ higher flood-defence walls is estimated to be $£(109-10-10-14-$ $10) \mathrm{M} / 0.92=£ 70.65 \mathrm{M}$. This then yields $£ 0.707 \mathrm{M}$ per one percent of flood protection by higher flood-defence walls.

The total costs to enhance flood-plain storage at Cononley Washlands and Holden Park are taken as $£ 35 \mathrm{M}$ (inclusive); this figure is based on FASI, which includes two very advanced adjustable weirs, higher defence walls and removal of a spit of land between the River Aire and the Aire-Calder navigation, and which costed £50M (Flood-Alleviation Scheme 2017).

Given the above, we define a basic scenario S0 providing flood protection against the 1:200-year return-period flood event by only $100 \%$ higher flood-defence walls. Scenario $\mathrm{S} 0$ is depicted in Fig. 9 as the 2m-deep square lake with the required FEV capacity of $V_{e}$; it has a $2150 \mathrm{~m}$ side-length and, given the height-to-width ratio of $\approx 0.1 \%$, subsequent depiction of the lake using only an aerial view is most convenient. Recall that the floodmitigation goal is to deal with the flood by elimination of the FEV, either by raising flood-defence walls, thus increasing $h_{T}$ and reducing the FEV, or by holding back flood volume, also reducing the FEV. In Fig. 9, we added the total costs and the costs per percentage of storage gained using double-headed arrows.

To illustrate our methodology, we will investigate four alternative scenarios, denoted by S1, S2, S3 and S4, and using data from Table 2, as follows:

- S1: the extra Calverley $8 \%$ flood-storage and reduced higher defence walls;

- S2: the extra Rodley 12\% flood-storage and further-reduced higher walls;

- S3: the extra Calverley and Rodley 14\% flood-storage and even-more-reduced higher walls;

- S4: the extra Cononley Washlands and Holden Park 50.4\% flood-storage sites and the most-reduced higher walls; these replace the Rodley and Calverley sites, which are most expensive per percentage storage gained, and which is also the case when Calverley and Rodley flood-storage is used in combination.

A visual comparison of the respective areas of each flood-mitigation measure S1 to $\mathrm{S} 4$ is found in Fig. 11, where the width of each flood-mitigation measure portrays a relative contribution because each strip has the side length of the entire square flood lake of uniform $2 \mathrm{~m}$-depth. The strips are augmented by the percentages of each floodmitigation measure as well as the total costs and costs per percentage gained. From Figs. 9 and 11, it becomes immediately clear that scenarios S0 and S4 are the cheapest. There are, however, some additional considerations to be taken into account. Scenario S0 does not reduce the $£ 14 \mathrm{M}$ required to update FASI to a 1:200-year return-period floodevent standard because the river water is not held back but, rather, flushed through the city of Leeds between higher flood-defence walls, that quickly transport the flood waters downstream. Significant floodwater storage as in scenario S4 does lower or may 

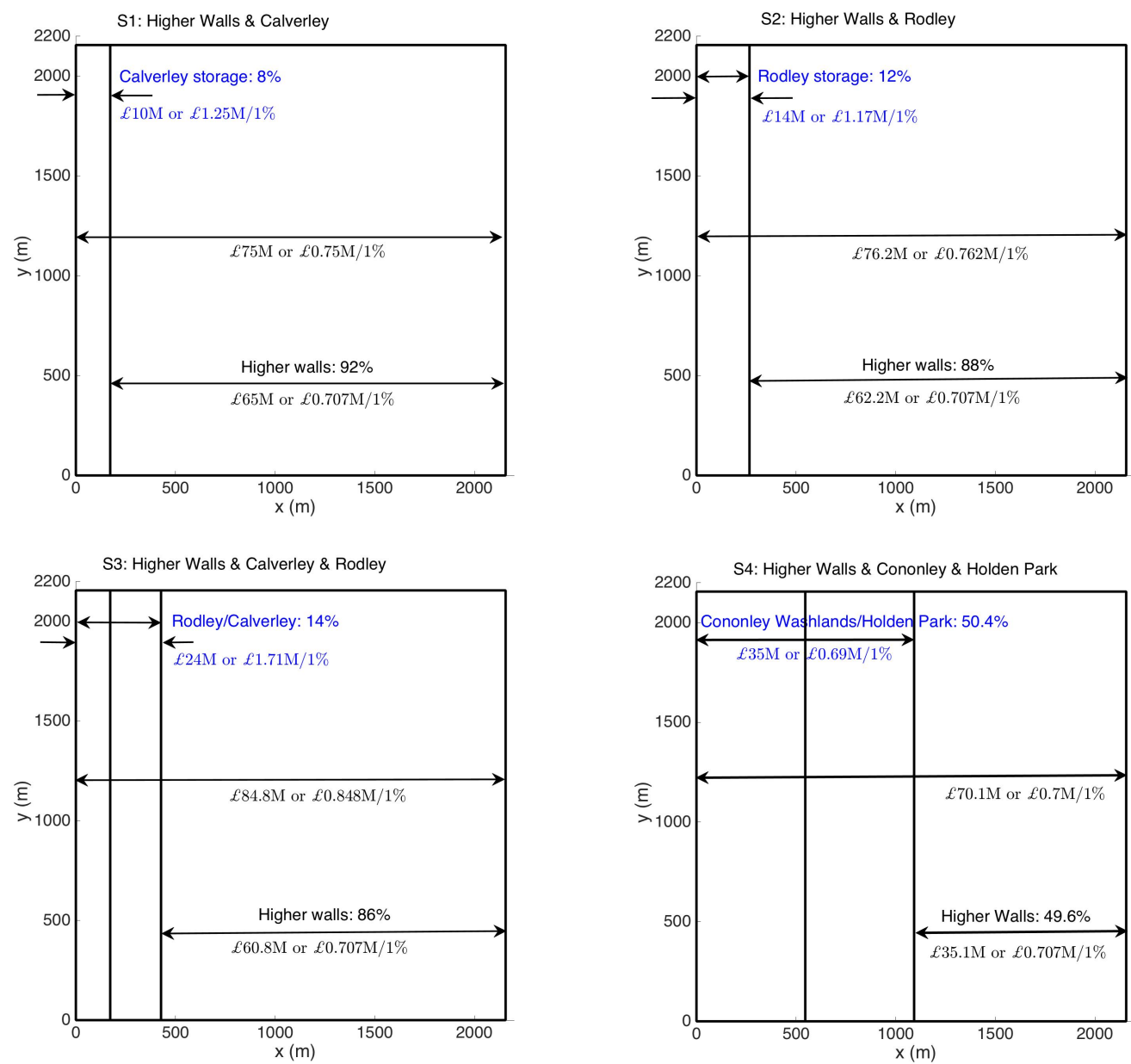

FIGURE 11. Flood-mitigation scenarios $\mathrm{S} 1$ to $\mathrm{S} 4$, with each flood-mitigation measure represented in a lake or "pie" chart, as lake area, and via the length of the (inverted) double-headed arrows. In addition, above or alongside the double arrow the total percentage of protection for each flood-mitigation measure is given; below the double arrow the costs and relative cost per percentage of flood mitigation is stated as well. Total costs are indicated under the double arrow spanning the entire width of the lake.

eliminate the need for updating FASI, the extent of which can best be assessed by further simulations of the flood hydraulics. Higher walls, such as those used in scenario S0, would protect only Leeds, whereas the enhanced flood-storage areas used in scenario S4 offer flood protection also further upstream, in other municipalities. Depending on the relative gains, it may be possible that it is advantageous to include the less cost-effective Rodley and Calverley storage sites as well. Furthermore, calculation of FEVs at multiple spatial locations along the River Aire and similar cost-benefit analyses as given above may provide a more comprehensive view of the optimal and cost-effective flood-mitigation strategy over the entire river catchment upstream of and including Leeds.

The overall conclusion of our analysis of $\mathrm{FASII}^{+}$is that our graphical presentation of the individual and overall effects of each flood-mitigation measure quantifies the reduction of the FEV, presented as a square flood lake of $2 \mathrm{~m}$ depth, directly combined with a costbenefit analysis. It offers a novel and thorough assessment of flood-mitigation strategies 

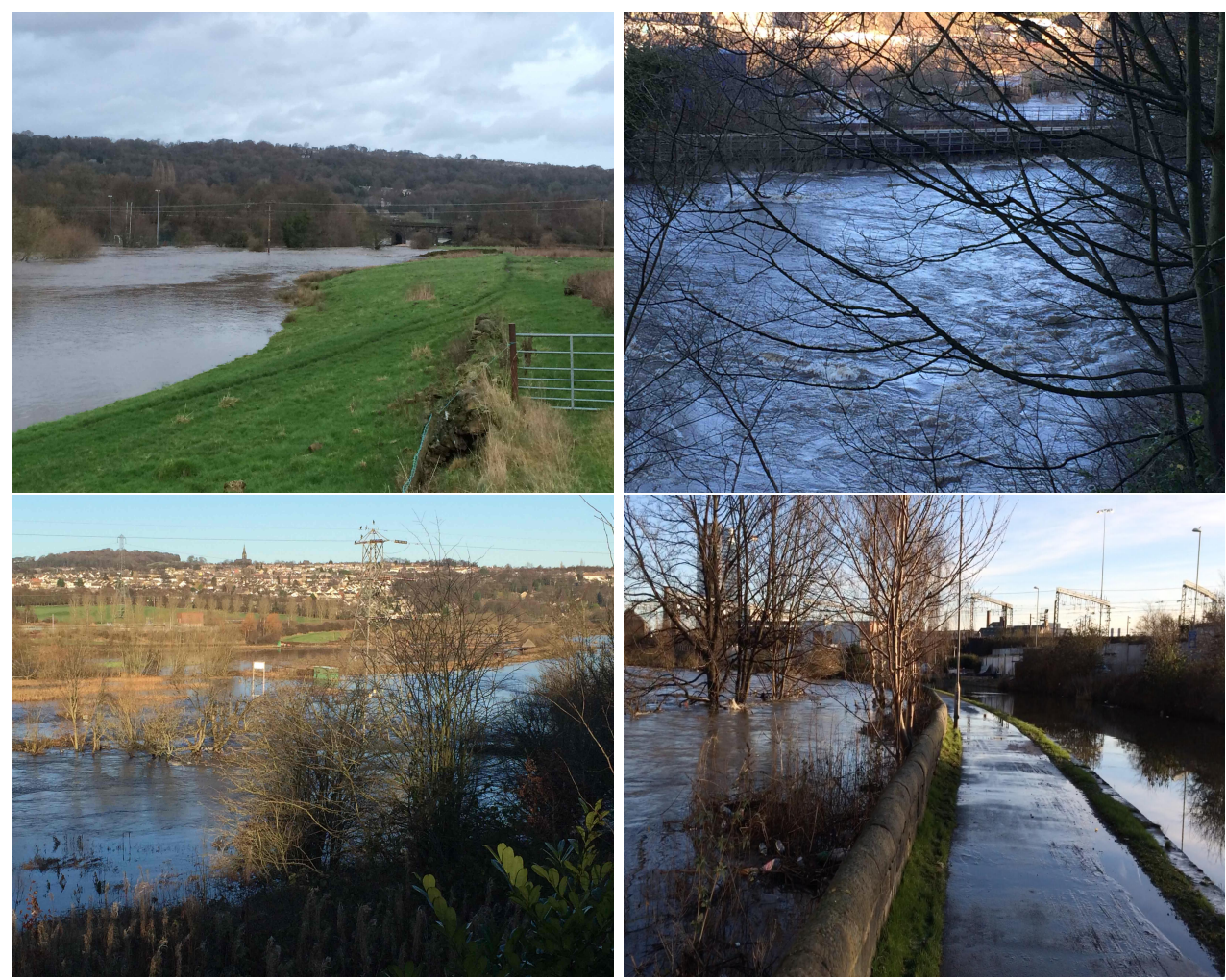

FiguRE 12. Evidence of extra or limited storage capacity above and beyond the Boxing Day 2015 flood levels for the River Aire; 1. top left: extra storage capacity downstream of Apperley Bridge both upstream of the Victorian railway bridge and downstream (26-12-2015 at 15:27:33); 2. top right: no extra storage capacity at Kirkstall The Forge, where now a shallow cut has been made of the sharp bend to alleviate flood risk on the train track visible in the photograph; 3. bottom left: extra storage on the Rodley nature reserve flood plain (27-12-2015 at 12:15:05); and, 4 . bottom right: downtown Leeds flooding just upstream of the station where the Leeds-Liverpool canal could have been modified as bypass of the hydraulic control point the "Dark Arches" under Leeds' train station (27-12-2015 at circa 13:20:53), shown in Fig. 2. Photos and video cut courtesy O.B.

that is simple to understand and adapt, and which can therefore further assist policy makers in determining near-optimal flood-mitigation strategies.

\section{Summary and discussion}

To facilitate the understanding and comparison of extreme flooding events, we have introduced the concept of flood-excess volume (FEV), which is the flood volume of river discharge minus the discharge $Q_{T}$ associated with a certain critical river-level threshold $h_{T}$ times flood duration. It is effectively the "area" under the flood hydrograph $Q(t)$ from $Q_{T}$ upward. Since FEV usually involves many millions of cubic metres, it is insightful to divide it by a depth of $2 \mathrm{~m}$, say, and take the square root: one then gets a square lake of $2 \mathrm{~m}$-deep with the same capacity as the FEV. Such a flood-excess lake offers a better sense of the size of a flood and whether or not that lake fits within the relevant river valley either as one piece or divided into sub-lakes over the course of the river. FEV is exactly the volume one wishes to eliminate in order to prevent flooding because its elimination would ensure that the in-situ river level stays below the chosen critical 
threshold $h_{T}$. Naturally there is variability of FEV with any chosen threshold $h_{T}$, but the latter level $h_{T}$ can neither be too small, otherwise there is no flood (damage), nor can it be above the peak level $h_{\max }$ of a flood event, since then FEV is by definition zero, i.e. $V_{e}\left(h_{\max }\right)=0$. To calculate FEV one generally must link measured river levels to discharge rates via rating curves; in so doing, one has to be careful because rating curves contain errors, which are often difficult to quantify. Several approximations for calculating rating curves, mean discharge and the FEV have been presented herein.

Subsequently, the FEV was estimated and calculated, both in averaged and detailed ways, for the Boxing Day 2015 floods of the River Aire at Armley in Leeds. For the River Aire, the FEV was calculated to be $V_{e}\left(h_{T}=3.9 \mathrm{~m}\right)=9.34 \mathrm{Mm}^{3}$ or, equivalently, the capacity of a square $2 \mathrm{~m}$-deep lake with a side-length of circa $2150 \mathrm{~m}$. The threshold of $h_{T}=3.9 \mathrm{~m}$ was carefully chosen as a flood threshold based on photographic and eyewitness evidence of the Boxing Day 2015 flood levels near Armley in combination with the river-level data measured.

FEV turns out to be extremely insightful in analysing the relative importance of floodmitigation measures proposed. Particularly useful is to ask and to answer the following question: what fraction of the chosen FEV, i.e., $V_{e}\left(h_{T}\right)$, is reduced by a particular floodmitigation measure or policy? This provides a welcome visualisation and interpretation that is comprehensible to scientists and non-scientists alike, including policy makers, the public and flood practitioners. For the sake of clarity, it is useful to express that fraction as the part of the relevant flood-excess lake of a particular depth. Hence we can express flood-mitigation measures visually and clearly as pieces of this square flood-excess lake. The corresponding costs of each flood-mitigation measure can be presented in unison in a lake-based visualisation, alongside the respective fraction of each flood-mitigation measure, thus leading to a clear and visual cost-benefit analysis for facilitating decisionmaking.

To illuminate the FEV analysis, we have applied it to several hypothetical floodalleviation scenarios (denoted by $\mathrm{FASII}^{+}$), inspired by the existing Leeds' FloodAlleviation Scheme II (FASII), and augmented with cost and flood-storage volume estimates that have so far been absent from the public domain. FASII aims to provide flood protection against a flood event with a 1:200-year return period upstream of Leeds' railway station and, in extension of Flood-Alleviation Scheme I (FASI), of which construction finished in 2017 and which offers protection to a flood with a 1:100-year return period downstream of the railway station. Five $\mathrm{FASII}^{+}$scenarios have been compared via a FEV cost-benefit analysis with flood-mitigation measures involving a combination of: flood-defence walls of various heights; a series of enhanced flood plains (at different locations and of different sizes); natural flood management, and; an update of FASI to a 1:200-year return-period flood. Such a FEV cost-benefit analysis provides a rational and advanced way to juxtapose and choose between flood-mitigation scenarios. It lends itself much better than detailed calculations or verbal statements, for dissemination of the effects of flood-mitigation measures proposed, in an understandable yet quantitative manner. For our hypothetical $\mathrm{FASII}^{+}$, scenario $\mathrm{S} 4$, with its use of large storage volumes upstream of Leeds as well as some higher flood-defence walls in Leeds, was found to be most advantageous in terms of flood mitigation and cost. Scenario S4 will have additional benefits for communities upstream of the Leeds' municipality and a minimal need to update FASI because floodwaters therein are partially held upstream of Leeds.

FEV is exactly the volume one wishes to eliminate in order to prevent flooding. In $\mathrm{FASII}^{+}$, several flood-mitigation approaches were assessed and each approach could in essence be expressed as a fraction of the FEV, visually represented as a part of the 
square flood-excess lake. Now extra flood-plain storage can, for example, be created by using dynamic weirs or sluice gates, to enhance and control the flood volume (for modern dynamic flood control, see Breckpot (2013) and Breckpot et al. (2013)), and a discovery that has been made explicit herein is that only the available flood-plain volume counts. This aspect is rarely recognised in practice, and indeed it is not accounted for in the existing FASII (Leeds Executive Board 2017). The proposed storage site at, for example, Rodley, had an estimated available flood-plain volume of $1.1 \mathrm{Mm}^{3}$ based on analysing wall-height information in FASII: this is only half the size of the quoted total flood-plain volume of $2.2 \mathrm{Mm}^{3}$, while a quick ballpark estimate based on photographic and floodmap evidence led to an estimated available flood-plain volume of $0.7 \mathrm{Mm}^{3}$. If one wants to protect against a flood with, e.g., a 1:200-year return period, then one has to realise that the flood-plain is already partially filled with floodwater in the absence of any weir, meaning that that the reference flood-storage volume comprising this floodwater is of course not available for extra flood storage. That reference storage volume needs to be subtracted from the total flood-storage volume in order to obtain the available floodstorage volume. For lower-return-period floods, the available flood-storage volume will of course be larger. As stated above, this concept of available flood-storage volume is related to the concept of available potential energy used as a diagnostic in meteorology (Lorenz 1955; Shepherd 1993) and it seems worthwhile to explore this analogy in the future.

Furthermore, it is important to stress that the use of FEV does not replace the need for performing detailed model calculations in certain situations. It does, however, provide immediate guidance to refine such calculations a priori or, as an important practical alternative, replace such calculations in cases where insufficient computational resources are available.

Finally, there are several possible extensions of our analysis, listed as follows:

- FEV can be used to diagnose detailed hydraulic-flow calculations a posteriori; rather than using a measured flood hydrograph, one can first compute a reference-flood hydrograph and an associated FEV (or a range of such volumes for a range of thresholds), and then express calculations of scenarios with various flood-mitigation measures relative to this reference-flood hydrograph as $(100 \%)$ reductions of the associated FEV; such an approach can also be explored in a probabilistic manner by using ensemble calculations for a distribution of reference-flood hydrographs with different return periods, with FEVs calculated and compared at various critical spatial locations;

- FEV can be used as a complementary way of classifying flood events; flood hydrographs can be narrow, high and low-volume or broad, relatively high and high-volume, each with vastly different FEVs; for flood mitigation it is meaningful and therefore of interest to reclassify return periods for river floods with sufficiently high peak levels in terms of FEV rather than in terms of only river-peak levels; this will, of course, be meaningful for only floods with peaks surpassing certain threshold levels $h_{T}$;

- FEV can play a central role in defining a new protocol to optimise the assessment of flood-mitigation scenarios, including a cost-benefit analysis; it may additionally prove beneficial in certifying such a protocol in flood-mitigation handbooks.

\section{Acknowledgments}

We are indebted to the Environment Agency in Yorkshire for providing data of Armley river gauges and information on rating curves for these sites. This work grew out of EPSRC's Living with Environmental Change (LWEC) UK network "Maths Foresees" (led by O.B.) as well as meetings within Sarah Dance's EPSRC Data Sci- 
ence Fellowship "Data Assimilation for the REsilient City" (DARE, with O.B. as coI). Part of the material and ideas presented have appeared in preliminary form in publicly available presentations of meetings and Industrial Study Groups of "Maths Foresees": see, e.g., www1.maths.leeds.ac.uk/mathsforesees/leeds2018.html and http://www.turing-gateway.cam.ac.uk/event/tgmw41/programme.

\section{REFERENCES}

Akers, B. \& Bokhove O. 2008 Hydraulic flow through a contraction: multiple steady states. Phys. Fluids 20, 056601.

Bokhove, O., Kelmanson, M.A., \& Kent, T. 2018 On using flood-excess volume to assess natural flood management, exemplified for extreme 2007 and 2015 floods in Yorkshire. Archived at https://eartharxiv.org

Breckpot, M. 2013 Flood control of river system with Model Predictive Control; the River Demer as a case study. PhD Thesis, KU Leuven, Belgium.

Breckpot, M., Agudelo, O. M., \& De Moor, B. 2013 Flood control with Model Predictive Control for river systems with water reservoirs. J. Irrigation and Drainage Engineering 139, 532-541.

BUSINESS INSIDER 2017 http://www.businessinsider.com/how-much-rain-hurricane-harvey-hasproduced-2017-8

Coles, S. 2001 An Introduction to Statistical Modeling of Extreme Values. Springer Series in Statistics 14, Springer, 209 pp. Springer.

Environment Agency 2016 Hydrology of the December 2015 Flood in Yorkshire. April 2016 report of Environment Agency.

Environment Agency Leeds 2016 River Aire at Armley, rating change report August 2016.

Environment Agency October 2017 Working with Natural Processes - Using the evidence base to make the case for Natural Flood Management. https: //www.gov.uk/government/uploads/system/uploads/attachment_data/file/654435/ Working_with_natural_processes_using_the_evidence_base.pdf

Environment AgEnCy JANuARY 2018 The costs of the winter 2015 to 2016 floods. https://www.gov.uk/government/uploads/system/uploads/attachment_data/ file/672088/costs_of_the_winter_floods_2015_to_2016_summary.pdf and https://www.gov.uk/government/uploads/system/uploads/attachment_data/file/ 672087/Estimating_the_economic_costs_of_the_winter_floods_2015_to_2016.pdf

Flood-Alleviation Scheme I, OCT. 2017 https://www.gov.uk/government/news/50-millionflood-defence-scheme-opens-in-leeds

GUARDIAN $25^{\text {th }}$ JUNE 2007 https://www.theguardian.com/uk/2007/jun/26/topstories3. weather

Guardian, 26 ${ }^{\text {th }}$ December 2015 Severe flooding in Britain prompts Boxing Day evacuations - as it happened. https://www.theguardian.com/environment/live/2015/dec/26/ severe-flood-warnings-in-the-north-prompt-boxing-day-evacuation-fears

Hodgkins, G.A., Whitfield, P.H., Burn, D.H., Hannaford, J., Renard, B., Stahl, K., Fleig, A.K., Madsen, H., Mediero, L., Korhonen, J., Murphy, C., Wilson, D. 2017 Climate-driven variability in the occurrence of major floods across North America and Europe. J. Hydrology 552, 704-717.

Intergovernmental Panel on Climate Change 2013 Fifth Assessment Report, Climate Change 2013. Summary for Policymakers, pp. 5 and 7, and Technical Summary, pp $40 \mathrm{ff}$.

Leeds City Council 2018 Leeds Flood-Alleviation Scheme Phase II. www.leeds.gov.uk/fas

LeEds Executive BOARD meEting 2017 Decision details 04-12-2017 (pdfs at bottom of page). http://democracy.leeds.gov.uk/ieDecisionDetails.aspx?ID $=45047$

Lorenz, E.N. 1955 Available potential energy and the maintenance of the general circulation. Tellus 7, 157-167.

SANDERSOn,M. 2010 Changes in the frequency of extreme rainfall events for selected towns and cities. Met Office "Ofwat" report. https://www.ofwat.gov.uk/wp-content/uploads/ 2015/11/rpt_com_met_rainfall.pdf

Shepherd. T.G., 1993 A unified theory of available Potential Energy. Atmos.-Ocean. 31, 1-26. 
Trigg, M.A., Bates, P.D., Wilson, M.D., Schumann,G. \& Baugh,C., Floodplain channel morphology and networks of the middle Amazon River. Water Resources Research 48, 10504.

UK Government 2018 Flood probablity maps. https://flood-map-for-planning.service. gov.uk/summary/419052/437681 https://flood-map-for-planning.service.gov.uk/ summary/419052/437681.

Vionnet, C.A., Tassi, P.A., Rodriguez, L.B. \& Ferreira, C.G. 2006 Numerical modelling of the catastrophic flooding of Santa Fe city, Argentina. Int. Journal of River Basin Management 4.

West Yorkshire COMBINED AUthority 2016 Leeds city region flood review report. December 2016. https://www.westyorks-ca.gov.uk/media/2532/ leeds-city-region-flood-review-report-executive-summary-final.pdf

\section{Appendix A. NFM and upper bounds on the flood-mitigation effects of tree planting}

The River Aire catchment has approximate area $A_{T}=1000 \mathrm{~km}^{2}$, roughly aligned in a $60 \times 6.5 \mathrm{mi}^{2}$ rectangle (see wikipedia). Trees are typically planted less than $60 \mathrm{ft} \approx 18.18 \mathrm{~m}$ apart (see wikipedia), so $243 \mathrm{k}$ trees cover an extra area of circa $A_{t}=80 \mathrm{~km}^{2}$, which is indeed the aforementioned $8 \% \approx A_{t} / A_{T}$ of tree covering in the catchment area, cf. FASII's NFM (Leeds Executive Board 2017, §3.2.3). Estimated rainfall and peakdischarge increase due to climate change vary but let us assume it is $24 \%$, to the extent that there appears to be no significant increase of extreme rainfall and peak discharge for flood events with a return period of 1:100 years or more, while there is such an increase for flood events with lower return periods. We refer to Hodgkins et al. (2017) and references therein; also observe that in Sanderson (2010) the climate projections display great uncertainty for the most extreme case with a 1:100-year return period considered therein, implying that these sources are not necessarily contradicting another. Accordingly there is, with the present data available, no need to mitigate against climatechange extreme events for Leeds, but NFM measures will be useful to protect against flooding from less-severe flood events impacting infrastructure and dwellings further upstream of any of the more advanced, proposed flood-mitigation measures in FASII. Tree planting will most likely occur in the upper catchment above Kildwick and, since the upper catchment comprises roughly a third of the catchment area, trees would thus cover about $3 \times 8 \%=24 \%$ of this area in the upper catchment. The peak flows at Kildwick and Armley were respectively $163 \mathrm{~m}^{3} / \mathrm{s}$ and $344 \mathrm{~m}^{3} / \mathrm{s}$, cf. Environment Agency (2016). Assuming an upper-bound case by taking 100\% absorption of rainfall due to tree planting, trees would then reduce the peak flow at Kildwick by $24 \%$ to about $124 \mathrm{~m}^{3} / \mathrm{s}$ and at Armley to about $305 \mathrm{~m}^{3} / \mathrm{s}$; these estimates are based on a linear scaling that can be improved by using the Kildwick and Armley river-gauge data. From Fig. 8(b), it is clear that this upper bound on the discharge reduction via the tree-planting measure can at best only partially alleviate the FEV of $V_{e}\left(h_{T}=3.9 \mathrm{~m}\right)$ because we see that the threshold discharge is $Q\left(h_{T}=3.9 \mathrm{~m}\right)=219 \mathrm{Mm}^{3}$ (and the above rate of $305 \mathrm{~m}^{3} / \mathrm{s}$ is still much higher). Moreover, given that an upper bound on the water absorption was used, and given the uncertainties surrounding NFM's effectiveness for extreme rainfall events, it is best to use this NFM measure only to (partially) offset increased run-off due to climate change, as intended in FASII. The FASII plans are ultimately clear about the role of NFM to mitigate climate-change effects only, but only because no quantification - which would admit a specific cross-examination - of its effectiveness is provided; by contrast, there is a clear quantification of flood protection against a 1:200-year flood for the four cases in Table 2 involving higher flood-defence walls. 